\title{
Variations in Physiological Responses of Participants During Different Stages of an Immersive Virtual Environment Experiment
}

\author{
Andrea Brogni ${ }^{1}$, Vinoba Vinayagamoorthy ${ }^{1}$, Anthony Steed ${ }^{1}$, Mel Slater ${ }^{1,2}$ \\ ${ }^{1}$ Department of Computer Science, University College London - London, UK \\ ${ }^{2}$ ICREA, Departament de LSI, Universitat Politécnica de Catalunya - Barcelona, Spain \\ \{a.brogni | v.vinayagamoorthy | a.steed | m.slater\}@cs.ucl.ac.uk
}

\begin{abstract}
This paper presents a study of the fine grain physiological responses of participants to an immersive virtual simulation of an urban environment. The analysis of differences in participant responses at various stages of the experiment (baseline recordings, training, first half and second half of the urban simulation) are examined in detail. It was found that participants typically show a stress response during the training phase and a stress response towards the end of the simulation of the urban experience.

There is also some evidence that variations in the level of visual realism based the texture strategy used was associated with changes in mental stress.
\end{abstract}

\section{Categories and Subject Descriptors}

I.3 [Computer Graphics]: Three-Dimensional Graphics and Realism - Virtual reality; J.4 [Computer Applications]: Social And Behavioral Sciences - Psychology

\section{General Terms}

Human Factors

\section{Keywords}

Immersive Virtual Environments, Physiology, Human Response, Evaluation

\section{INTRODUCTION}

Today's virtual environment (VE) systems cannot produce sensory output that has fidelity close to physical reality. For example, it remains costly to obtain real-time global illumination for visual outputs, although recent research have started to address this problem ( see [8] in this volume). Similarly, realtime generation of sound and full body haptics remain problematic. Despite the relatively poor quality of the stimuli being created, evidence suggests that people do tend to exhibit behaviours and responses as

Permission to make digital or hard copies of all or part of this work for personal or classroom use is granted without fee provided that copies are not made or distributed for profit or commercial advantage and that copies bear this notice and the full citation on the first page. To copy otherwise, to republish, to post on servers or to redistribute to lists, requires prior specific permission and/or a fee.

VRST'06, November 1-3, 2006, Limassol, Cyprus.

Copyright 2006 ACM 1-59593-321-2/06/0011 ...\$5.00. if the situation were real; it is as if basic minimal cues are operational that provide sensory data that is sufficient for the human perceptual system to respond to them as if they were real. Perhaps the most compelling demonstrations of virtual environments come from simulations of stressful situations. Meehan et al. [12] used physiological monitoring to demonstrate that participants behave with an appropriate physiological stress response when subjected to a stressful virtual environment.

In this paper, we expand the use of physiological monitoring to look at the response of the participant in more general situations that do not contain stimuli designed to be shocking. The motivation is that by observing more of the physiological response of the participant we may better interpret subjective ratings and observations of the participants' performance. The main idea is to use the physiological response as a technique for the evaluation of the stress level during virtual experiences. During different phases of the same virtual experience, the participant may have different responses to the environment and perceive the experience in different mental and physical states.

This work exploits knowledge about how the nervous system reacts to novel situations. In our study, we recorded physiological signals from 40 participants during a immersive experience in a virtual street environment that contained virtual people walking through the street. We then analyzed the recorded reactions of the participants during different time segments, in particular before and during the virtual experience, in order to determine the psychophysiological impact of the VE on the participant. We utilized the physiological responses of the participant as an evaluative method in determining the level of stress experienced by the participant during the experience. The experiment also investigated the impact of variations in the scene across two conditions: texture quality and visual realism of the virtual people. This aspect is reported in [23].

In the following sections we discuss an overview of the most common techniques used in measuring participants' reactions in a VE and some further background on the use of heart-rate and heart-rate variability measures. Then, we describe the design of the experiment, we outline the methods used in analyzing the physiological data followed by discussion of the findings. Finally, we briefly present intentions for future work based on the current results.

\section{HUMAN REACTIONS IN VE}

\subsection{Presence}

The propensity of subjects to act within virtual environments as if the stimuli were real in some sense, has been called presence. There are several definitions of and scientific approaches to pre- 
sence in the literature [4] [14]. Witmer et al. [25] defined presence as "the sense of being there (in a virtual environment), even when one is physically situated in another place (lab)". In this paper we adopt a recent definition of presence introduced by Sanchez Vives and Slater [13], according to which "Presence occurs when there is a successful substitution of real sensory data by computer generated sensory data, and ... the person responds to the virtual stimuli as if they were real". The response should be considered at multiple levels ranging from physiological through to conscious behavioural and cognitive responses. In this paper we focus only on physiological responses, in particular heart rate and heart rate variability.

There are numerous physiological measurements that could be recorded during a study using non invasive devices. The most common are heart rate (HR), skin conductance (otherwise known as galvanic skin response or GSR) and skin temperature, electroencephalogram (EEG) and electrocardiogram (ECG). Wiederhold at al. [24] used physiological responses to analyze the behaviour of "phobic" and "non-phobic" individuals in a virtual environment. Meehan [11] used physiological responses to measure how believable the experience of being at the edge of a virtual pit was. The hypothesis was that if the people were present, the virtual pit would have been able to evoke physiological responses similar to those of a corresponding real environment. They also noted that HR and GSR measure the arousal of the individual, therefore might only be used when such arousal is intrinsic to the task, e.g. fear.

Other experiments have shown that GSR and HR could be used as objective measures in monitoring participant reactions to virtual environments [7]. Recent evidence suggests a relationship between events during a virtual reality experience and physiological responses. In particular, breaks in presence (BIP) [17] are associated with detectable physiological responses in GSR and ECG recordings $[15,16]$.

\subsection{Heart Rate and Heart Rate Variability}

The relationship between mental and bodily processes originates in the central nervous system (CNS) which acts as an "interface" between the external world and the internal organs [9]. In particular, it is the autonomic nervous system (ANS) that controls the activities of the internal organs, such as heart contraction and rate, sweat gland activity, skin temperature and many other visceral functions. Andreassi [2] reports on the relationship between physiological manipulations and corresponding physiological responses. In stressful situations the body responds in a variety of ways such as increasing the HR and electrodermal activity (EDA), decreasing the skin temperature or inducing rapid and shallow breathing and so on. These responses are generally modulated by the Sympathetic Nervous System (SNS) and lead to the body experiencing what is commonly termed stress. When the causative conditions change and the body can recover, the nervous system reduces the body stress level in an attempt to revert the body back to it's normal state using the Parasympathetic Nervous System (PNS).

$\mathrm{HR}$ is a measure of the times per minute the heart contracts. The typical range is between 60 and $90 \mathrm{bpm}$ (beats per minute). Using the mean HR to evaluate the level of stress and presence in different conditions can give an idea of the overall stress of the experience, but it cannot determine what this stress has been caused by [12]. Therefore, we need to use a wider range of analysis on the HR, to understand the stress and its causes.

Heart Rate Variability (HRV) is computed from the raw ECG signal and it can then be used to describe the physiological behaviour of the individual under a specific situation. This form of analysis is particulary useful in understanding event related experiences [1]. HRV is influenced, at different frequencies, by the ANS and in particular by the activities of the Parasympathetic and Sympathetic Nervous Systems (PNS and SNS). The low frequency components (LF) of the HRV $(0.04 \mathrm{~Hz}-0.15 \mathrm{~Hz})$ are modulated by both the PNS and SNS. The high frequency components $(\mathrm{HF})$, between the range of $0.15 \mathrm{~Hz}$ and $0.4 \mathrm{~Hz}$, are mainly modulated by the PNS. The ratio LF/HF describes the balanced behaviour of the SNS and PNS. In situations of mental stress, a rise in the LF components and a decrease in the HF components is observed leading to an increase in the LF/HF ratio. During dynamic exercise, or any activity involving physical stress, an increase of the HR is noticed but this is accompanied with a stable level of the HF components.

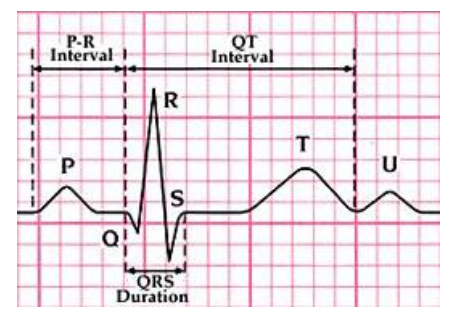

Figure 1: QRS complex in the HR signal.

A typical ECG signal has a repeated wave with 6 peaks (named $\mathrm{P}, \mathrm{Q}, \mathrm{R}, \mathrm{S}, \mathrm{T}$ and $\mathrm{U}$ in fig. 1). The R-peak is the highest and the reference for the heart beat, but usually we refer to the QRS complex, that includes the previous and the following small peaks. The RR interval is the distance in time between to following QRS complexes, those represent the time it takes for depolarization of the ventricles. The NN signal is the normal to normal beat time distance. Generally the RR and NN intervals are taken to be the same however in some individuals extrasystoles (premature contractions of the heart chamber) are recorded. The NN values reflect analysis of cleaned up ECG data from the extrasystoles. The changes in the $\mathrm{RR}$ (or NN) interval can be described in the time and frequency domains, and they determine the HRV.

\section{THE EXPERIMENT}

\subsection{Experimental Goals}

The object of this study was to examine if there were variations in the stress level during a VE experience. We were interested in whether physiological responses could useful as a tool for evaluating what reactions the volunteers had and whether or not they had event-related stress during the experience. As part of the experimental design we were interested in investigating whether there needs to be consistency between the levels of realism of the different elements within a scene, in particular buildings and characters (see 3.3 for the details).

\subsection{Scenario}

The VE explored by the participants was a replica of a street with virtual people walking around. The layout of the street can be seen in Figure 2.

During the experiment, at any one time, 8 out of set of 16 different virtual characters were seen walking along the street, avoiding any collision with each other and with the participant. Depending on the condition of the experiment, the characters were cartoon-like or semi photorealistic, as shown in Figure 3.

\subsection{Independent Variables}

The two main factors in the study were the texture quality of the environment and the visual aspect of the virtual characters: 


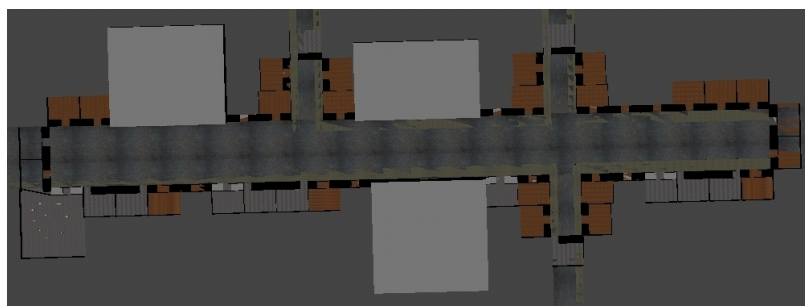

Figure 2: Top view of the street.

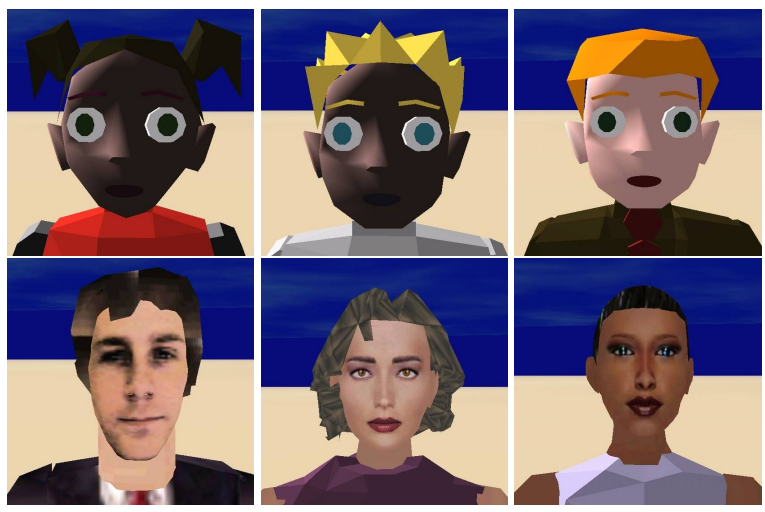

Figure 3: Cartoon(above) and Realistic (below) Avatars.

\section{Texture quality of the environment}

- Repetitive (20 textures, repeated twice on different buildings),

- Non-repetitive (40 textures, none repeated).

Visual aspect of the characters

- Cartoon-like in aspect and form,

- Visually semi photorealistic and humanoid-like.

In Section 4 we refer to the four conditions as:

- HH - High level textures (non repeated) and High level (realistic) avatars

- LH - Low level textures (repeated) and High level (realistic) avatars

- HL - High level textures (non repeated) and Low level (cartoony) avatars

- LL - Low level textures (repeated) and Low level (cartoony) avatars

\subsection{Dependent Variables}

During the experiment, the volunteers were asked to signal (by pressing a button of the joystick) whenever they had a BIP. After the experiment, the SUS [17] and the ITC-SOPI [10] questionnaires were completed by the participants as subjective measures of presence. In addition the participant's behavior during the experience was recorded on video tape along with positional data obtained from the tracking sensors.

Three physiological signals have been recorded: respiration, GSR and ECG. Respiration has been only used as a control signal during the recording, but no analysis has been planed on it. GSR analysis is on going. This paper is only on the analysis of the ECG with respect to HRV. ECG was sampled at $256 \mathrm{~Hz}$. Measurements of HRV parameters as discussed in Section 4.3 were performed using the g.BSanalyze biosignal software package by Guger Technology [6].

\subsection{Explanatory Variables}

A demographics questionnaire was administered about age, gender, occupation, language proficiency, experience in computer games, programming and virtual reality.

\subsection{The System}

Participants were placed inside a CAVE-like system [3], where the VE was projected. The CAVE-like system used was a Trimension ReaCTor, consisting of three $3 \mathrm{~m} \times 2.2 \mathrm{~m}$ solid acrylic walls and a $3 \mathrm{~m}$ x $3 \mathrm{~m}$ floor. It was powered by a $300 \mathrm{MHz}$ Silicon Graphic Onyx 2 with 8 MIPS R12000 processors, 8GB RAM and 4 InfiniteReality2 graphics pipes. The participants wore lightweight CrystalEyes stereo glasses, tracked by a wireless Intersense IS900 system, with 6DOF ultrasonic sensors. The participants were able to navigate in the VE using a wireless navigation joystick-like device, with direction of movement determined by the pointing direction. The frame rate of the simulation was around $45 \mathrm{~Hz}$.

The software used was a derivative of Distributed Interactive Virtual Environment (DIVE) version 3.3x [5, 19] which was ported to support spatially immersive systems [19]. DIVE is an internetbased multi-user virtual reality system in which participants can navigate in a shared $3 \mathrm{D}$ space.

The ProComp Infinity, an eight channel device by Thought Technologies LTD [21], was used for physiological data acquisition.

\subsection{Procedure}

Forty individuals were recruited for the experiment. The participants were distributed equally amongst the four conditions that resulted: five males and five females for each group. Three different experimenters helped with the running of the studies. In any one session, two of the three experimenters guided the participant through the study. The roles played by the experimenters were randomized in order to minimize the impact of experimenter bias on findings.

After initial greetings, the participants were asked to complete a consent form after going through an information sheet. The participant was then asked to answer an online questionnaire containing a series of demographic questions. After this, the participants were trained to record breaks in presence following the method described in [15], but the results of this analysis are reported in [23] and a different study that examined the relationship between breaks in presence and physiological responses is reported in [16].

The participant was introduced to the ReaCTor and fitted with various devices: tracking sensors, shutter glasses and physiological sensors. Initially the participant was asked to stand still for about 90 seconds in the dark in order to record a baseline for the physiological signals in a relaxed and non-active state. After the baseline session, the VE appeared on the projection screen, at which point on of the experimenters explained the use of the joystick-like navigation device. The participant was asked to practice navigation in a training room (see Figure 4). The training room is a virtual room in which free-standing single-digit 3D numbers were displayed floating over the floor. The participant was asked to move through the training room by following the number sequentially from 1 to 9 until he/she felt confident. Finally, the participant was reminded to signal any transitions from the VE to the real world (breaks in 
presence) by pressing buttons on the joystick-like device. Then the participant was asked to stand in front of a virtual door leading to the virtual street. At this point the participant was instructed to complete an exploration task in the street once the virtual door opened.

The virtual experience lasted around four minutes for each participant. After three minutes of virtual experience, the colour of the sky turned dark to signal the end of the experience, and the participants returned to the starting point in the virtual environment following instructions given to them before the study. Each study lasted about 30 minutes in total and each participant was paid $£ 5$ (pounds sterling).

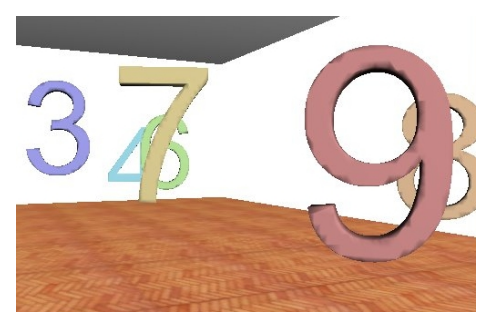

Figure 4: Training room with numbers.

\section{ANALYSIS AND RESULTS}

\subsection{Introduction}

In this Section we focus on the analysis of the ECG; in particular the HRV analysis in both the time and frequency domains. The aim was to detect differences in the physiological responses of the participants, in the different segments: baseline, training and the actual experiment segments. The state of the participants during different segments of the study might give an interesting insight into the stress level experienced by the participants during the experience. For instance the experience while recording the baseline signal, quite a relaxing situation, is different to their experience in the training segment. The physical activity required of the participant changes through the different segments; for instance the baseline segment requires the least amount of effort in comparison to the others.

\subsection{Data Pre-Processing}

For each participant, the baseline segment lasted for 90 seconds and was used as a reference for comparison with the other segments. The training segment was not limited to a fixed time because it was important to let the participants start the main part of the experiment only when they felt comfortable enough. The exploration of the street lasted 3 minutes. The maintenance of a stringent timeline during the experiment was key to obtaining useable physiological data. Conducting HRV analysis requires similar time slots (segments) in order to be able to make valid comparisons. From the complete recording of each participant we extracted four 90 second segments. The first of these segments was the baseline. The second segment consisted of the last 90s of the training session, in order to avoid including the dramatic change induced by the transition from the baseline period, which required no activity, to the training period that required significant activity. The experience in the street was divided into two different segments, the first 90 seconds and the last 90 seconds. The whole experience was thus treated as four segments: baseline (B), training (T), first 90 seconds of the experiment (E1) and second 90 seconds of the experiment (E2).

\subsection{HRV Algorithm}

The RR time series were resampled at a frequency of $2 \mathrm{~Hz}$. Then the power spectrum of the resampled time series was estimated using the Burg method [20] of order 15. The RR sequence was detrended and a Hanning window was applied prior to the spectrum estimation. The FFT length was 128 with an overlap of 64 and a sampling frequency of $2 \mathrm{~Hz}$. Once all the measures are estimated, we have applied a sign test for paired couples, comparing the values for the different four time segments (B, T, E1 and E2). The Matlab ${ }^{1}$ tools used for the HRV analysis was the g.BSanalyze by Guger Technology [6]. This analysis has allowed us to evaluate the different mental and physical stress levels between the 90 second segments.

\begin{tabular}{|c|l|}
\hline meanRR & mean of the RR interval (ms) \\
\hline SDNN & standard deviation of the NN interval (ms) \\
\hline maxRR and minRR & max and min values in the RR interval (ms) \\
\hline MinMaxRR & difference between MaxRR and MinRR (ms) \\
\hline MeanHR and SDHR & mean and standard deviation of the HR (bpm) \\
\hline SDANN & $\begin{array}{l}\text { stand.dev. of the average NN interval calculated over } \\
\text { short periods }\end{array}$ \\
\hline SDNNindex & $\begin{array}{l}\text { mean of e.g. 1 min stand.dev. of NN intervals calcu- } \\
\text { lated over total recordig lenght }\end{array}$ \\
\hline SDSD & stand.dev. of successive NN differences (ms) \\
\hline RMSSD & $\begin{array}{l}\text { square root of the mean squared difference of suc- } \\
\text { cessive NN intervals (ms) }\end{array}$ \\
\hline NN50 & $\begin{array}{l}\text { number of intervals of successive NN intervals } \\
\text { greater than 50 ms }\end{array}$ \\
\hline pNN50 & $\begin{array}{l}\text { NN50 divided by the total number of NN intervals } \\
(\%)\end{array}$ \\
\hline
\end{tabular}

Table 1: Time domain parameters evaluated for the HRV.

\subsection{Results}

In this Section we present the results related to the HRV analysis on the ECG signals. The sign tests show the significant variations for the values in the comparisons between the four time segments:

- $\mathrm{B} / \mathrm{T}$ - sign test comparing the values in the baseline and in the training segments,

- $\mathrm{T} / \mathrm{E} 1$ - sign test comparing the values in the training and in the first segment of the experiment,

- E1/E2 - sign test comparing the values between the two segments of the experiment,

In Table 2, the raw results with the sign tests for all the 40 participants are shown, without gender or experimental factorials. We can observe that comparing the baseline segment to the training segments, there is an arising in the LF/HF ratio and no change in the HR measures, showing an increase in the mental stress level. When the participants started the experiment segment, the LF/HF ratio decreases along with the stress level and the physical activity. In the second segment of the experiment, both the stress level and the physical activity increases. In addition, there are significant differences in terms of HR, between the training segment and the first segment of the experiment. There is a reduction in the mean HR. However between the first and the second segments of the experiment, there is an increase in the mean HR.

The analysis indicates that there are different mental and physical stress levels in the different time segments of the experiment. The mental stress seems to increase at the beginning of the virtual experience, during the training, then it decreases at the beginning of

${ }^{1}$ www.mathworks.com 
the actual experiment, and it increases again at the end of the experiment. The physical stress seems being higher during the training and second half of the experiment.

The next step in the analysis was to evaluate different reactions between the experimental factorials (see section 3.3). In Table 3, only the significant $\mathrm{p}$ values of the sign test are shown. The correspondent HRV variables are shown in Table 4. We can see significant $\mathrm{p}$-values indicating a significant variation in mean HR between the training segment and the first experiment segment, in all the conditions but HL (non repeated textures and non realistic avatars), where the SDHR decreases. An important aspect is the p-values for the rate LF/HF between training and the first segment of the experiment, where the value decreases in the conditions with non repeated textures (HL and $\mathrm{HH}$ ).

The general effect of having less physical activity during the experiment segment, comparing with the training segment, is also confirmed between the conditions. The lower mental stress once they start the actual experiment is only verified for a subset of the conditions, showing a variation due to the level of realism of the avatars.

The last step in the analysis was to split the data according to the gender, to see whether or not females and males react in a different way during such an experience. In none of the four conditions where there strongly significant differences between males and females.

\subsection{Discussion}

The HRV variations provide good indications for stress level during real experiences [1]. One of the goals of this study was to examine similar techniques in relation to VR experiences, and to see if HRV analysis could be applied in order to determine whether or not the participants had stress during the experience.

In the above analysis, we can observe how the mental and physical stresses changed during the virtual experience. When comparing the baseline to the training segment, we notice an increase in the mental stress level. This finding has many possible explanations. The participants find themselves in a novel situation, undergoing the first or one of few VE experiences of their life, so they become curious, not knowing what is to come and whether they would be able to adequately carry out their side of the experiment.

During the training segment, the participants are asked to move, learn how to manage with the technology, but when the experiment starts, observations from reviewing the recordings reveal that most participants stand in the middle of the ReaCTor space while using the joystick on the wand to navigate to the ends of the virtual street. This requires much less physical activity than was required in the training phase. During the actual experiment, we notice a decrease in the mental stress or in the physical activity. The participant may

\begin{tabular}{|rrrrclll|}
\hline \multicolumn{1}{|c|}{ values } & \multicolumn{1}{c|}{ p values } \\
\hline $\mathbf{B}$ & $\mathbf{T}$ & $\mathbf{E 1}$ & $\mathbf{E 2}$ & & B/T & T/E1 & E1/E2 \\
69.015 & 67.488 & 70.681 & 69.679 & mean RR & 0.4296 & 0.0000 & 0.0166 \\
40.675 & 46.113 & 38.074 & 35.000 & SDNN & 0.2682 & 0.0064 & 0.1539 \\
89.206 & 91.132 & 86.837 & 88.254 & mean HR & 0.4296 & 0.0000 & 0.0166 \\
5.060 & 6.022 & 4.582 & 4.401 & SDHR & 0.0166 & 0.0000 & 0.4296 \\
23.684 & 25.354 & 23.520 & 22.083 & RMSSD & 0.6358 & 0.2682 & 0.0064 \\
23.782 & 25.455 & 23.618 & 22.174 & SDSD & 0.6358 & 0.2682 & 0.0064 \\
8.375 & 7.975 & 7.200 & 7.225 & NN50 & 1.0000 & 0.2430 & 0.8601 \\
7.254 & 6.523 & 6.210 & 6.128 & pNN50 & 1.0000 & 0.1877 & 0.6076 \\
1.727 & 2.111 & 2.147 & 1.922 & SDANN & 0.0385 & 0.8746 & 0.1539 \\
17.139 & 16.840 & 16.394 & 15.607 & SDNN index & 0.6358 & 0.8746 & 0.0064 \\
68.490 & 72.955 & 65.351 & 69.823 & LF norm & 0.0807 & 0.0166 & 0.0385 \\
23.713 & 16.437 & 23.110 & 19.935 & HF norm & 0.0064 & 0.0007 & 0.0385 \\
4.671 & 6.299 & 4.606 & 5.650 & LF div HF & 0.0166 & 0.0007 & 0.0385 \\
\hline
\end{tabular}

Table 2: HRV values (the mean all over the participants) and $p$ values of the sign tests for the 40 participants. p-values of the sign tests below 0.05 are significant.

\begin{tabular}{|rrrcrrr|}
\hline & LL & & & & LH & \\
\hline B/T & T/E1 & E1/E2 & & B/T & T/E1 & E1/E2 \\
1.000 & 0.021 & 0.344 & mean HR & 0.754 & 0.021 & 0.109 \\
0.109 & 0.021 & 1.000 & SDHR & 0.754 & 0.109 & 0.109 \\
1.000 & 0.070 & 1.000 & NN50 & 0.754 & 1.000 & 0.727 \\
1.000 & 0.039 & 1.000 & pNN50 & 0.754 & 1.000 & 0.508 \\
0.754 & 1.000 & 0.109 & LF norm & 0.754 & 0.344 & 0.109 \\
0.754 & 1.000 & 0.109 & HF norm & 0.109 & 0.109 & 0.109 \\
0.754 & 1.000 & 0.109 & LF div HF & 0.344 & 0.109 & 0.109 \\
\hline & HL & & & & HH & \\
\hline B/T & T/E1 & E1/E2 & & B/T & T/E1 & E1/E2 \\
0.109 & 0.344 & 0.109 & mean HR & 0.754 & 0.002 & 1.000 \\
0.021 & 0.021 & 1.000 & SDHR & 1.000 & 0.109 & 1.000 \\
1.000 & 0.344 & 1.000 & NN50 & 0.508 & 0.508 & 1.000 \\
1.000 & 0.344 & 1.000 & pNN50 & 0.508 & 0.508 & 1.000 \\
0.344 & 0.344 & 0.754 & LF norm & 0.021 & 0.021 & 0.344 \\
0.344 & 0.021 & 0.754 & HF norm & 0.109 & 0.021 & 0.344 \\
0.344 & 0.021 & 0.754 & LF div HF & 0.109 & 0.021 & 0.344 \\
\hline
\end{tabular}

Table 3: Sign tests for the different factors, but no gender discrimination (HH means non repeated textures and realistic avatars; LH means repeated textures and realistic avatar, and so for the others). p-values of the sign tests below 0.05 are significant.

\begin{tabular}{|c|c|c|c|c|c|c|c|c|}
\hline \multicolumn{5}{|c|}{ LL } & & \multicolumn{2}{|l|}{ LH } & \\
\hline B & $T$ & E1 & E2 & & $\bar{B}$ & $T$ & E1 & E2 \\
\hline 84.27 & 85.18 & 81.49 & 82.36 & mean HR & 86.28 & 87.25 & 82.84 & 83.78 \\
\hline 4.35 & 5.25 & 3.64 & 3.48 & SDHR & 4.41 & 5.85 & 4.30 & 3.74 \\
\hline 5.80 & 5.60 & 3.50 & 3.30 & NN50 & 6.80 & .90 & 7.30 & 6.50 \\
\hline 5.02 & 4.53 & 2.90 & 2.78 & pNN50 & 6.64 & 5.63 & 6.65 & 6.23 \\
\hline 72.54 & 72.88 & 69.16 & 72.81 & LF norm & 76.47 & 76.28 & 69.19 & 76.64 \\
\hline 20.13 & 16.34 & 21.17 & 17.64 & F n & 16.40 & 11.26 & 16.87 & 12.64 \\
\hline 4.60 & 5.62 & 4.80 & 6.36 & LF div $\mathrm{HF}$ & 5.52 & 9.41 & 6.41 & 8.24 \\
\hline \multirow{2}{*}{\multicolumn{5}{|c|}{ HL }} & \multicolumn{3}{|c|}{$\mathrm{HH}$} & \\
\hline$\overline{\mathbf{B}}$ & $\mathbf{T}$ & & $\overline{E 2}$ & & $\overline{\mathbf{B}}$ & $\mathbf{T}$ & E1 & $\overline{E 2}$ \\
\hline 91.44 & 94.89 & 91.33 & 92.94 & mean & 94.83 & 97.20 & 91.68 & 93.94 \\
\hline 5.44 & 6.93 & 5.16 & 5.48 & SDH & 6.05 & 6.06 & 5.22 & 4.90 \\
\hline 9.10 & 10.30 & 7.00 & 8.50 & NN & 11.80 & 9.10 & 11.00 & 10.60 \\
\hline 7.64 & 8.71 & 6.25 & 6.82 & pNN & 9.72 & 7.22 & 9.04 & 8.68 \\
\hline 65.96 & 73.83 & 66.96 & 67.76 & F n & 58.98 & 68.83 & 56.09 & 62.08 \\
\hline 25.57 & 17.30 & 23.47 & 24.06 & $\mathbf{F n}$ & 32.76 & 20.85 & 30.93 & 25.41 \\
\hline 5.44 & 5.73 & 4.24 & 3.95 & LF div HF & 3.12 & 4.43 & 2.97 & 4.05 \\
\hline
\end{tabular}

Table 4: HRV variables for the different factors, but no gender discrimination (HH means non repeated textures and realistic avatars; LH means repeated textures and realistic avatar, and so for the others). 
become familiar with the technology and more confident therefore they start to relax in the virtual environment. Figure 5 outlines the main variations in the stress level.

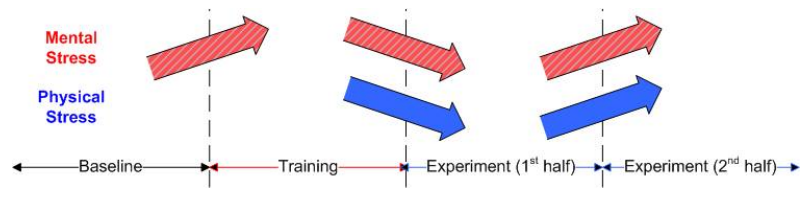

Figure 5: The variations of the stress during the virtual experience.

One of the interesting aspects of the analysis is in the differences between the first and second experiment segments. The physical activity increases and even the mental stress increases. Therefore, in the second half of the experiment, the participants start moving more and also seem to become more stressed. One possibility is that the participants start to notice more technical problems and the spatial limits of the environment. Another possibility is that something about the "social interaction" disturbs them - since the virtual characters uncharacteristically ignore them completely, which could be disturbing. There is some evidence of this reported in [22]. For example, in a preliminary analysis of behaviour in a pilot experiment using the same model, we have observed participants signalling BIPs when the notice that textures are repeated in the environment. This and similar faults are more likely to be detected the longer the participant is exposed to the Virtual Environment [18]. As a consequence it may be possible that the mental stress, during the complete experience of a virtual reality system, is high at the beginning when the participants do not know what to expect. However once the participants become more relaxed, observation of faults in the model may become an important factor, and also of course the content of the simulation would itself be significant.

In [23], there is the presence analysis related to this experiment, based on presence questionnaires and BIPs. The authors made a presence measurement during the whole virtual experience, expecting the level of the textures and the behavior of the avatars to be the key determinants of the presence level. In particular, they suggest that the perception of the realism in the character behaviour is positively correlated to the level of presence. The level of the texture (repeated or no-repeated) was also important for the differen level of presence across the conditions: the condition with repeated textures and realist avatars (LH in this paper) produced the lower reported presence response.

In this paper, we shifted the attention of the analysis to the level of stress, but still using conditions based on key features in the environment. The correlation between the level of mental stress and the quality of the texture has been confirmed and could be correlated to the level of presence. In particular, the conditions with no-repeated texture resulted the ones with lower level of stress and higher level of presence. Also the hypothesis about the lack of social interaction in the avatar, as a disturbing factor, finds a similar hypothesis in the presence analysis, where the perceived character behaviour is important.

This suggests that one feature that might be investigated in future is whether faults in the environment cause BIPs and lead to a higher level of stress. In this experiment we do not have large enough numbers of BIPS to study this in detail. The number of BIPs across the subjects is 124 , where 55 were during the first 90 seconds and 69 during the last 90 seconds. A t-test over the data gives a p-value of 0.088 . This means that there is a difference and the number of
BIP increased in the second half of the experiment, but in this case the variation was not significant but it only gives an indication for the next studies. Further studies and investigations are required to explore the implications of these results.

\section{CONCLUSION AND FUTURE WORK}

In our study, we recorded the ECG signals for all 40 participants before and during the experience of a virtual urban simulation. We ran a HRV analysis in time and frequency domains on separate $90 \mathrm{~s}$ time segments selected from these recordings.

Firstly, the analysis indicated that being introduced into an immersive virtual environment creates a stressful situation for the participants, compared to the pre-experience baseline session. The stress level decreases once they get inside the more natural-looking and engaging environment that was designed for the experiment. We hypothesize that this was because the participants began to get used to the new technology and that they felt more comfortable as time passed by. In the last segment of the experience, the participants tended to become more stressed perhaps due to a lack of interest in the environment, a mundane explorative task, or because the technological limits in the system become more apparent. Although our explanations of the change in stress levels can only be considered hypotheses, the fact that we can detect differences is very promising and suggests that these types of time-varying responses should be taken into account when analysing participant behavior in such VEs.

Secondly, comparing between the condition in the experiment design, we found that the condition with non repetitive textures, that is the more visually realistic environment, was one that most influenced the mental stress of the participant. Although a preliminary result, it suggests that the effects of visual fidelity are having an impact on much higher levels of response.

HRV analysis has proved itself an interesting tool for the evaluation of mental and physical stress in participants during a virtual reality experience. It has highlighted that people respond respond with stress to a training period, which, for this experiment, reduced once the main study started. This suggests that in future experiments people should be allowed to continue training until they report being very comfortable with the controls and understand their tasks. The study has also generated a hypothesis - that the longer an experiment continues there is greater chance for participants to become aware of problems with the system, which itself may be a stress inducer.

\section{ACKNOWLEDGEMENT}

This work was supported by the UK project EQUATOR Interdisciplinary Research Collaboration (EPSRC Grant GR/N15986/01). Thanks to David Swapp for support on the UCL ReaCTor, to Marco Gillies for the PIAVCA software and its integration into DIVE and to Ashwin Beeharee for help in running the experiment.

\subsection{References}

\section{REFERENCES}

[1] AA.VV. Heart rate variability: Standards of measurement, physiological interpretation and clinical use. European Heart Journal, 17:354-381, 1996. Task Force of the European Society of Cardiology and the North American Society of Pacing and Electrophysiology. Guideline of the Working Groups on Arrhythmias and Computers in Cardiology of the ESC and the North American Society of Pacing and Electrophysiology. 
[2] J. J. Andreassi. Psychophysiology: Human Behavior and Physiological Response. Lawrence Erlbaum Associates, London, UK, 4th edition, 2000.

[3] C. Cruz-Neira, D. Sandin, and T. DeFanti. Surround-screen projection-based virtual reality: The design and implementation of the CAVE. ACM Computer Graphics, 27(2):135-142, 1993.

[4] J. V. Draper, D. B. Kaber, and J. M. Usher. Telepresence. Human Factors, 40(3):354-375, 1998.

[5] E. Frécon, G. Smith, A. Steed, M. Stenius, and O. Stahl. An overview of the coven platform. Presence: Teleoperators and Virtual Environments, 10(1):109-127, February 2001.

[6] C. Guger, G. Edlinger, R. Leeb, G. Pfurtscheller, A. Antley, M. Garau, A. Brogni, D. Friedman, and M. Slater. Heart-rate variability and event-related ecg in virtual environments. In Presence 2004: The 7th Annual International Workshop on Presence, pages 240-245, 2004.

[7] D. P. Jang, I. Y. Kim, S. W. Nam, B. K. Wiederhold, M. D. Wiederhold, and S. I. Kim. Analysis of physiological rensonse to two virtual environments: Driving and flying simulation. CyberPsychology \& Behavior, 5(1):11-18, 2002.

[8] P. Khanna, I. Yu, J. Mortensen, and M. Slater. Presence in response to dynamic visual realism:a preliminary report of an experiment study. In VRST, Cyprus, 2006. ACM.

[9] J. W. Kimball. Biology. William C. Brown, Boston, MA, U.S.A, 6th edition, 1994.

[10] J. Lessiter, J. Freeman, E. Keogh, and J. Davidoff. A cross-media presence questionnaire: The itc-sense of presence inventory. Presence: Teleoperators and Virtual Environments, 10(3):282-298, 2001.

[11] M. Meehan. Physiological Reaction as an Objective Measure of Presence in Virtual Environment. $\mathrm{PhD}$ thesis, University of North Carolina at Chapel Hill, USA, 2001.

[12] M. Meehan, B. Insko, M. Whitton, and J. Frederick P. Brooks. Physiological measures of presence in stressful virtual environments. In Proceedings of ACM - Transactions on Graphics, volume 21, pages 645-653. ACM SIGGRAPH, 2002.

[13] M. V. Sanchez-Vives and M. Slater. From presence to consciousness through virtual reality. Nature Neuroscience, 6(4):8-16, April 2005.
[14] M. Slater. Presence and the sixth sense. Presence: Teleoperators and Virtual Environments, 11(4):435 - 439, August 2002.

[15] M. Slater, A. Brogni, and A. Steed. Physiological responses to breaks in presence: A pilot study. In Presence 2003: The 6th Annual International Workshop on Presence, Aalborg, Denmark, 2003.

[16] M. Slater, C. Guger, G. Edlinger, R. Leeb, G. Pfurtscheller, A. Antley, M. Garau, A. Brogni, A. Steed, and D. Friedman. Analysis of physiological responses to a social situation in an immersive virtual environment. Presence: Teleoperators and Virtual Environments, 15(5), 2006.

[17] M. Slater and A. Steed. A virtual presence counter. Presence: Teleoperators and Virtual Environments, 9(5):413-434, October 2000.

[18] A. Steed, A. Brogni, and V. Vinayagamoorthy. Using breaks in presence to identify usability issues. In Proc. of the 11th International Conference on Human Computer Interaction, Las Vegas, July 2005. HCI International 2005.

[19] A. Steed, J. Mortensen, and E. Frécon. Spelunking: Experiences using the dive system on cave-like platform. In B. Frohlicj, J.Deisinger, and H.-J. Bullinger, editors, Virtual Environments, pages 153-164. Springer-Verlag, 2001.

[20] P. Stoica and R. L. Moses. Introduction to Spectral Analysis. Prentice-Hall, 1st edition, 1997.

[21] Thought Technology Ltd., Montreal, Canada. ProComp+: Encoder \& Sensor User Instruction - tech Note 009, 2002.

[22] V. Vinayagamoorthy. User Responses to Virtual Humans in Immersive Virtual Environments. $\mathrm{PhD}$ thesis, University College London, University of London, London, UK, 2006.

[23] V. Vinayagamoorthy, A. Brogni, M. Gillies, M. Slater, and A. Steed. An investigation of presence response across variations in visual realism. In The 7th Annual International Presence Workshop, October 2004.

[24] B. K. Wiederhold, D. P. Jang, S. I. Kim, and M. D. Wiederhold. Physiological monitoring as an objective tool in virtual reality therapy. CyberPsychology \& Behavior, 5(1):77-82, 2002.

[25] B. G. Witmer and M. J. Singer. Measuring presence in virtual environments: A presence questionnaire. Presence: Teleoperators and Virtual Environments, 7(3):225 - 240, 1998. 